\title{
From critical care nephrology to critical care blood purification
}

\author{
Rongli Yang ${ }^{1}$, Xiukai Chen ${ }^{2}$, Suwei $\mathrm{Li}^{3}$, Xiaoting Wang ${ }^{4}$, Dawei Liu ${ }^{4}$ \\ 'Department of Critical Care Medicine, Dalian Municipal Central Hospital Affiliated of Dalian Medical University, \\ Dalian 116033, Liaoning Province, China; \\ ${ }^{2}$ Pittsburgh Heart, Lung, and Blood Vascular Medicine Institute, Department of Medicine, University of \\ Pittsburgh School of Medicine, Pittsburgh, PA, USA; \\ ${ }^{3}$ Department of Critical Care Medicine, The First Affiliated Hospital of Dalian Medical University, Dalian 116011, \\ Liaoning Province, China; \\ ${ }^{4}$ Department of Critical Care Medicine, Peking Union Medical College Hospital, Peking Union Medical College \\ and Chinese Academy of Medical Sciences, Beijing 100730, China
}

\section{INTRODUCTION}

Since Claudio Ronco and Rinaldo Bellomo proposed Critical Care Nephrology (CCN) more than 20 years ago, ${ }^{[1]}$ international CCN organizations such as Acute Dialysis Quality Initiative (ADQI) and Acute Kidney Injury Network (AKIN) have been established successively. The definition and staging of acute kidney injury (AKI) were unified, ${ }^{[2-4]}$ a large number of academic studies related to AKI were completed, and a series of guidelines or consensus on AKI and Continuous Renal Replacement Therapy (CRRT) were published. ${ }^{[4-8]}$ The vigorous development of CCN has enabled patients with severe AKI to get standardized treatment, and at the same time, CRRT has been well promoted in intensive care units (ICUs). However, blood purification in the ICU is not limited to Renal Replacement Therapy (RRT); there are many extrarenal indications for CRRT, and severely ill patients may also need other blood purification techniques such as apheresis or adsorption. Hiromichi Suzuki and Hiroyuki Hirasawa of Japan described blood purification in ICU in their book Acute Blood Purification published in 2010. ${ }^{[9]}$ Claudio Ronco and others have successively proposed the concepts of Multiple Organ Support Therapy (MOST) and Extracorporeal Organ Support (ECOS), both of which use extracorporeal blood purification technology to provide multiple organ support treatment for critically ill patients with Multiple Organ Dysfunction
Syndrome (MODS). ${ }^{[10,11]}$

With the rapid development of critical care medicine in China, the application of blood purification in ICUs is also increasing. In order to differ from traditional blood purification, we collectively refer to all blood purification treatments implemented in the ICU for critically ill patients as Critical Care Blood Purification (CCBP). After years of theoretical development and clinical practice, CCBP has developed not only as a technology, but as a scientific system with distinctive features in basic theories, clinical application principles and methods, and treatment implementation rules. We have systematically explained CCBP. ${ }^{[12,13]} \mathrm{We}$ gave a scientific definition of CCBP and summarized the four major characteristics of CCBP that are different from traditional blood purification.

\section{THE DEFINITION AND RESEARCH CONTENTS OF CRITICAL CARE BLOOD PURIFICATION}

CCBP is not just a set of technologies, but an interdisciplinary subject of critical care medicine and blood purification. CCBP is a science that studies the correlation and changes laws between the body's internal environment and critical illnesses and studies as well as uses blood purification technologies to treat critical illnesses under the guidance of critical medicine theory. ${ }^{[12]}$ 
CCBP not only needs to research and apply various blood purification techniques, but also needs to conduct in-depth research on the internal environment disorders caused by various severe diseases under the guidance of critical care medicine theory. Of course, the development of CCBP is inseparable from the support of technology. CCBP treatment often requires a variety of blood purification techniques or technique combinations, and these technologies are also the research objects of CCBP. CCBP techniques mainly include hemofiltration, hemodialysis, plasmapheresis, adsorption technologies, extracorporeal membrane oxygenation (ECMO), extracorporeal carbon dioxide removal $\left(\mathrm{ECCO}_{2} \mathrm{R}\right)$, and some combination techniques. CCBP is gradually breaking through the traditional "organ" and "replacement" levels, paying more attention to the internal environment and its regulation technology, and has its own characteristics and mode of action.

As a new science, CCBP itself is constantly developing, and its research content will continue to deepen and enrich. In an ICU, those who need blood purification treatment include not only patients with AKI, but also critically ill patients with other organs or systems involved. From the perspective of research scope, CCN is a major branch of CCBP. In addition to the kidneys, CCBP can also cover multiple organs or systems such as the liver, pancreas, heart, lung, immune system, nervous system, and muscles. At present, the research on blood purification treatment in these fields is not as in-depth as the research on renal supportive treatment. There is little unified standard and norm for blood purification treatment in these critically ill patients. If the successful experience of $\mathrm{CCN}$ is copied to other CCBP subspecialties, it will improve the prognosis of severely ill patients with other organ failures.

\section{THE MAIN CHARACTERISTICS OF CRITICAL CARE BLOOD PURIFICATION}

As an emerging interdisciplinary subject, CCBP has distinct characteristics, mainly in the following aspects:

\section{Critical care blood purification is inseparable} from the guidance of critical care medicine theory Critical care medicine has developed rapidly in recent years, enabling more and more critically ill patients to receive effective treatment. One of the main reasons that critical care medicine can improve the prognosis of patients is to emphasize the early detection and early intervention of severe diseases, as well as the concept of organ protection, such as early detection and treatment of shock, and lung protection strategies for acute respiratory distress syndrome. In addition, critical care medicine attaches importance to the study of the interaction between organs, such as cardiorenal syndrome, hepatorenal syndrome, and multiple organ dysfunction syndrome. Paying attention to the interaction between organs helps to balance a variety of treatment contradictions in the treatment process, thereby promoting the recovery of organ function and improving the prognosis. This treatment concept has also had a huge impact on CCBP. Taking acute renal failure and renal replacement therapy as examples, in order to achieve early detection and intervention of acute renal failure, the concept of "acute kidney injury" came into being; ${ }^{[2]}$ in order to protect the damaged kidney, the traditional approach of "renal replacement therapy" is gradually replaced by the concepts of "renal support therapy" and "multiple organ support therapy". ${ }^{[14]}$ Therefore, although the renal support treatment in CCBP is similar to the traditional RRT, there are obvious differences in the treatment indications and timing, as well as the method and dosage.

In addition, the volume adjustment range of critically ill patients is narrowed, and both insufficient volume and fluid overload will affect the prognosis. ${ }^{[15]}$ The progress of critical care medicine in the field of hemodynamics and fluid therapy also has a great impact on the volume management of CCBP. For example, when CRRT is performed on patients with unstable hemodynamics, only goal-directed treatment under the guidance of hemodynamics can achieve accurate and appropriate volume management, thereby improving the prognosis of those patients and their kidneys. ${ }^{[7]}$

\section{Personnel engaged in critical care blood purification must master the theories and skills of critical care medicine}

The fact that CCBP is inseparable from the guidance of critical care medicine theory also determines that the doctors who implement CCBP should have deep critical care medicine theory and skills, especially solid hemodynamic skills, in order to ensure the quality of CCBP. Whether CCBP is led by intensivists or nephrologists depends on the country or hospital. Which of the two forms is better or worse is still controversial. ${ }^{[16-19]}$ Vincent's research shows that under the premise of good multidisciplinary consultation, closed ICU formats with intensivist-led care can improve the prognosis of patients with acute renal failure. ${ }^{[16]}$ It shows that CCBP led by intensivists is a main direction for future development. Intensivists should strengthen their own learning and training and master the theory and skills of CCBP. Even for CCBP led by nephrologists, it should be ensured that they are trained in the theories and skills of critical care medicine. 
Critical care blood purification takes the internal environment of critically ill patients as the main research object and intervention target

Various internal environmental disorders can occur to critically ill patients. In addition to the volume, electrolyte and acid-base imbalance frequently seen in AKI, severe internal environmental disorders can also be caused by hepatotoxins in liver failure, pathogenic microorganisms, and cytokine storms in severe infections, pathogenic antibodies from autoimmune disease, exogenous drugs or poisons entering the body, hyperthermia caused by heat stroke, and so on. ${ }^{[20,21]}$

Using blood purification technology to rebuild homeostasis to maintain a good internal environment for organs and cells throughout the body will help restore organ function and improve prognosis. Traditional blood purification pays attention to the replacement of organ functions, while CCBP pays more attention to the correction of internal environmental disorders caused by various reasons such as organ dysfunction. Taking the internal environment disorder of critically ill patients as the target of CCBP intervention rather than just organ function is helpful to focus on the whole body and correctly determine the intervention timing, method, and dose of CCBP, reflecting the advantages of CCBP's systemic support. ${ }^{[5]}$

\section{Critical care blood purification has the characteristics of integration}

Due to the complexity and variability of critically ill patients, their internal environmental disorders are also diverse. Therefore, doctors are required to select appropriate blood purification techniques for patients and formulate individualized blood purification programs. Different from the relatively fixed treatment plan of traditional blood purification, CCBP often needs to flexibly select different blood purification techniques according to the changes of the patient's condition, showing the characteristic of integration. We divide the integration of CCBP into synchronous integration and sequential integration. Synchronous integration refers to the use of Integrated Blood Purification Technique (IBPT, that is, different blood purification principles or methods are integrated in the one composite technique) to treat critically ill patients, such as the use of Molecular Adsorption Recirculation System (MARS) to treat liver failure. Sequential integration refers to the use of different blood purification techniques one after another to treat the same critically ill patient. For example, patients with hepatorenal syndrome are treated with plasma exchange first, followed by CRRT.

In summary, CCBP, as a discipline that intersects with critical care medicine, cannot be developed without the guidance of critical care medicine theory. CCBP takes the internal environment of critically ill patients as the research object and intervention target. Due to the complexity and variability of critically ill patients, it is determined that the way of CCBP is not fixed, and different blood purification techniques need to be flexibly selected, showing a characteristic of integration. At present, the international $\mathrm{CCN}$ has made great achievements. With the deepening of CCBP's other subspecialty research and multidisciplinary cooperation, its concepts and technologies will inevitably be mastered by more clinical medical staff. The extensive and standardized application of CCBP will become another advantageous weapon that can significantly improve the prognosis of critically ill patients after mechanical ventilation.

\section{Research Funding}

None declared.

\section{Competing Interests}

Authors state no conflict of interest.

\section{REFERENCES}

1. Ronco C, Bellomo R. Critical care nephrology: the time has come. Nephrol Dial Transplant 1998; 13: 264-7.

2. Bellomo R, Ronco C, Kellum JA, Mehta RL, Palevsky P. Acute Dialysis Quality Initiative workgroup. Acute renal failure - definition, outcome measures, animal models, fluid therapy and information technology needs: the Second International Consensus Conference of the Acute Dialysis Quality Initiative (ADQI) Group. Crit Care 2004; 8: R204-12.

3. Mehta RL, Kellum JA, Shah SV, Molitoris BA, Ronco C, Warnock DG, et al. Acute Kidney Injury Network: report of an initiative to improve outcomes in acute kidney injury. Crit Care 2007; 11: R31.

4. Kellum JA, Lameire N, KDIGO AKI Guideline Work Group. Diagnosis, evaluation, and management of acute kidney injury: a KDIGO summary (Part 1). Crit Care 2013; 17: 204.

5. Ostermann M, Joannidis M, Pani A, Floris M, De Rosa S, Kellum JA, et al. Patient Selection and Timing of Continuous Renal Replacement Therapy. Blood Purif 2016; 42: 224-37.

6. Bagshaw SM, Chakravarthi MR, Ricci Z, Tolwani A, Neri M, De Rosa $\mathrm{S}$, et al. Precision Continuous Renal Replacement Therapy and Solute Control. Blood Purif 2016; 42: 238-47.

7. Murugan R, Hoste E, Mehta RL, Samoni S, Ding X, Rosner MH, et al. Precision Fluid Management in Continuous Renal Replacement Therapy. Blood Purif 2016; 42: 266-78.

8. Cerdá J, Baldwin I, Honore PM, Villa G, Kellum JA, Ronco C, et al. Role of Technology for the Management of AKI in Critically Ill Patients: From Adoptive Technology to Precision Continuous Renal Replacement Therapy. Blood Purif 2016; 42: 248-65.

9. Kaizu K, Inada Y, Kawamura A, Oda S, Hirasawa H. Current status of blood purification in critical care in Japan. Contrib Nephrol 2010; 166: 4-10.

10. Ronco C, Bellomo R. Acute renal failure and multiple organ dysfunction in the ICU: from renal replacement therapy (RRT) to multiple organ support therapy (MOST). Int J Artif Organs 2002; 25: 733-47. 
11. Ronco C, Ricci Z, Husain-Syed F. From Multiple Organ Support Therapy to Extracorporeal Organ Support in Critically Ill Patients. Blood Purif 2019; 48: 99-105.

12. Yang RL, Li SW, Chen XK. [Critical care blood purification: from technology to science]. Zhonghua Yi Xue Za Zhi 2017; 97: 2241-3.

13. Liu DW, Yang RL, Chen XK. Critical care blood purification (in Chinese). first edition. Beijing: People's Medical Publishing House 2017: 1-519.

14. Husain-Syed F, Ricci Z, Brodie D, Vincent JL, Ranieri VM, Slutsky AS, et al. Extracorporeal organ support (ECOS) in critical illness and acute kidney injury: from native to artificial organ crosstalk. Intensive Care Med 2018; 44: 1447-59.

15. Ronco C, Kaushik M, Valle R, Aspromonte N, Peacock WF 4th. Diagnosis and management of fluid overload in heart failure and cardio-renal syndrome: the "5B" approach. Semin Nephrol 2012; 32: 129-41.

16. Vincent JL. Critical care nephrology: a multidisciplinary approach. Contrib Nephrol 2007; 156: 24-31.

17. Flaatten H, Darmon M. A nephrologist should be consulted in all cases of acute kidney injury in the ICU: yes. Intensive Care Med 2017; 43: 874-6.
18. Kellum JA, Hoste E. A nephrologist should be consulted in all cases of acute kidney injury in the ICU: No. Intensive Care Med 2017; 43: 877-9.

19. Morgan D. Optimal Role of the Nephrologist in the Intensive Care Unit An Intensivist's Opinion. Blood Purif 2017; 44: 267-8.

20. Redant S, Hussein H, Mugisha A, Attou R, De Bels D, Honore PM, et al. Differentiating Hyperlactatemia Type A From Type B: How Does the Lactate/pyruvate Ratio Help. J Transl Int Med 2019; 7: 43-5.

21. Babazadeh A, Mohseni Afshar Z, Javanian M, Mohammadnia-Afrouzi M, Karkhah A, Masrour-Roudsari J, et al. Influenza Vaccination and Guillain-Barré Syndrome: Reality or Fear. J Transl Int Med 2019; 7: $137-42$.

How to cite this article: Yang R, Chen X, Li S, Wang X, Liu D. From critical care nephrology to critical care blood purification. J Transl Intern Med 2021; 9: 4-7. 